\title{
Duration of Cerebrospinal Fluid Drainage in Patients With Aneurysmal Subarachnoid Hemorrhage for Prevention of Symptomatic Vasospasm and Late Hydrocephalus
}

\author{
Yutaka HIRASHIMA, Masanori KURIMOTO, Nakamasa HAYASHI, \\ Kimiko UMEMURA, Emiko HORI, Hideki ORIGASA*, and Shunro ENDO \\ Department of Neurosurgery and *Division of Biostatistics, \\ Toyama Medical and Pharmaceutical University, Toyama
}

\begin{abstract}
The optimal duration of cerebrospinal fluid (CSF) drainage after acute aneurysm surgery is unclear. The association between the occurrence of symptomatic vasospasm or late hydrocephalus and the duration of CSF drainage was investigated using multiple logistic analysis in 95 consecutive patients with aneurysmal subarachnoid hemorrhage who underwent surgery within 72 hours after onset. The duration of drainage was significantly related to the occurrence of symptomatic vasospasm and late hydrocephalus. The cut-off values of the duration of drainage for preventing symptomatic vasospasm and late hydrocephalus were 11 days (adjusted odds ratio $0.347,95 \%$ confidence interval 0.135-0.889, $p=0.0274$ ) and 6 days (adjusted odds ratio 4.86, 95\% confidence interval 1.46-16.2, $p=0.0099$ ), respectively. Prevention of both symptomatic vasospasm and late hydrocephalus is not possible without additional procedures such as cisternal irrigation using fibrinolytic agents.
\end{abstract}

Key words: subarachnoid hemorrhage, cerebrospinal fluid drainage, symptomatic vasospasm, late hydrocephalus, logistic regression analysis

\section{Introduction}

Cerebral vasospasm remains an important cause of mortality and morbidity following subarachnoid hemorrhage (SAH) despite advances in the management of delayed cerebral ischemia using prophylactic calcium antagonists, ${ }^{14,18)}$ intrathecal fibrinolytic therapy, ${ }^{4,5,13,17)}$ and intravascular neurosurgical technique including transluminal angioplasty ${ }^{7)}$ and intraarterial infusion of agents. ${ }^{11)}$ The etiology and pathogenesis of vasospasm after $\mathrm{SAH}$ are not completely understood. Vasospasm may be induced by spasmogenic substances released from the subarachnoid clot, ${ }^{3,16)}$ so cerebrospinal fluid (CSF) drainage is commonly initiated during the acute stage of surgery for ruptured aneurysm to remove the residual clot and prevent cerebral vasospasm. Fibrinolytic agents such as urokinase and tissuetype plasminogen activator are also used to accelerate dissolution and removal of the clot. ${ }^{3,16)}$ However, increased incidence of cerebral infarction and late

Received June 24, 2004; Accepted November 22, 2004 shunt-dependent hydrocephalus have been observed following excessive drainage of CSF. ${ }^{1,9,12,15)}$ Meningitis and intracranial hemorrhage are also possible complications of CSF drainage. ${ }^{17)}$ No data is available about the duration of CSF drainage necessary after acute surgery to prevent the occurrence of vasospasm and late hydrocephalus.

The present study assessed the relationship between duration of CSF drainage after acute surgery and subsequent events of both types after adjusting for other factors.

\section{Subjects and Methods}

\section{Study population}

One hundred nineteen consecutive patients with SAH underwent aneurysm neck clipping for ruptured aneurysm at the Department of Neurosurgery, Toyama Medical and Pharmaceutical University, between January 1996 and December 2001. The exclusion criteria in this study included the following: patients who underwent surgery for aneurysm more than 72 hours after onset (12 patients), and interval between onset of SAH and ventriculope- 
Table 1 Summary of the characteristics of patients with and without symptomatic vasospasm after subarachnoid hemorrhage

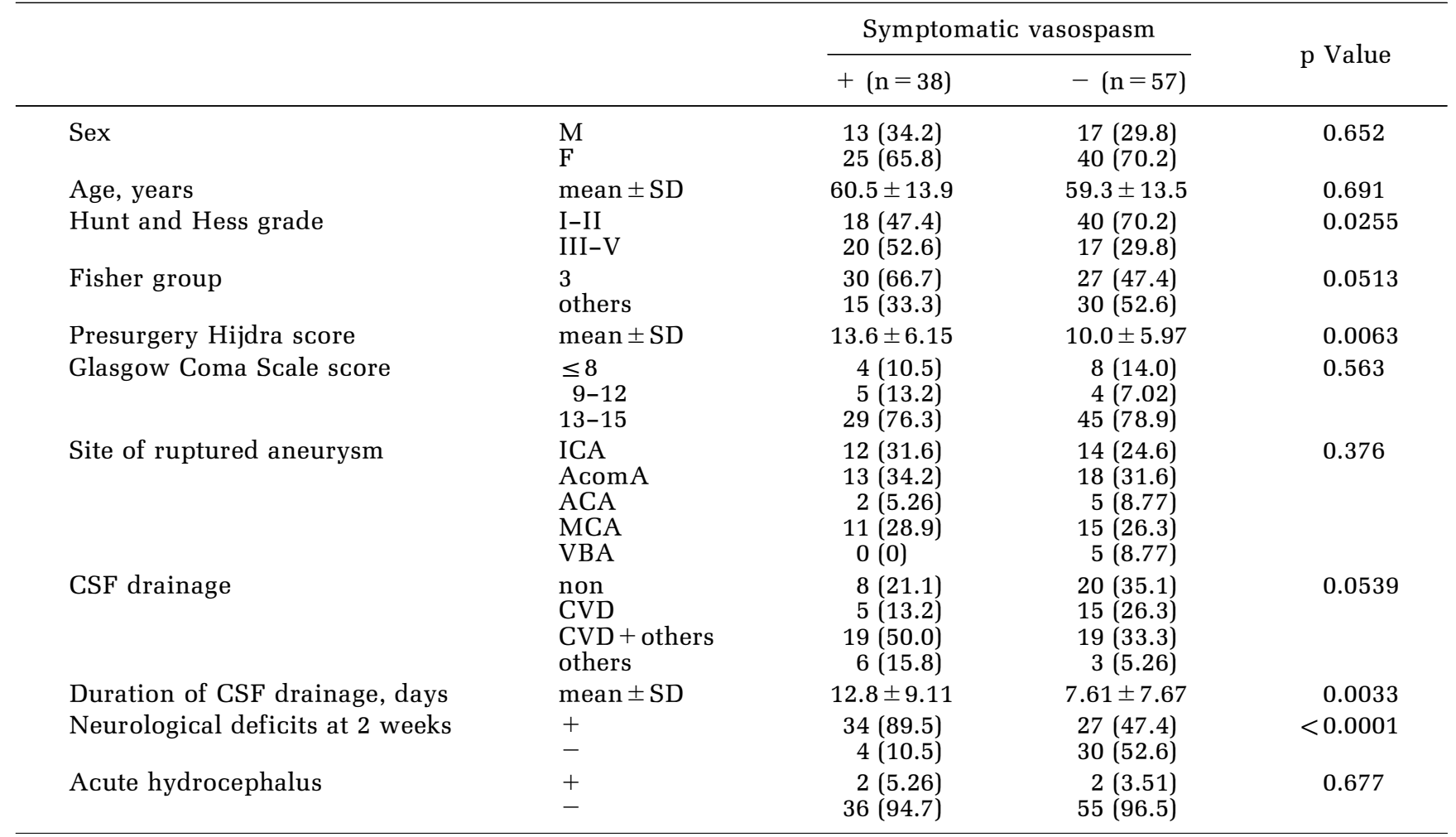

Figures in parentheses are percentages. ACA: anterior cerebral artery, AcomA: anterior communicating artery, CSF: cerebrospinal fluid, CVD: continuous ventricular drainage, ICA: internal carotid artery, MCA: middle cerebral artery, VBA: vertebrobasilar artery.

ritoneal (VP) shunting of $46.8 \pm 16.9$ days (mean \pm SD). All patients who died within 1 month of SAH (mean - SD) (11 patients) were excluded because the occurrence of late hydrocephalus could not be evaluated accurately. Finally, this study included 95 patients, 30 men and 65 women aged 24-86 years (mean 59.8 years). The clinical data for these 95 patients are summarized in Tables 1 and 2 .

\section{Data collection}

Age, sex, Hunt and Hess grade, ${ }^{10)}$ Fisher group, ${ }^{6)}$ and Glasgow Coma Scale score ${ }^{19}$ ) on admission, location of ruptured aneurysm, amount of SAH on admission according to the method of Hijdra, ${ }^{8)}$ duration of external drainage of CSF, acute hydrocephalus, symptomatic vasospasm, and neurological deficits at 2 weeks after SAH were assessed in each patient.

Hunt and Hess grade ${ }^{10)}$ and Glasgow Coma Scale score $^{19)}$ on admission were evaluated by the primary neurosurgeons. Brain computed tomography (CT) was performed immediately after admission using an YCT-900S (Toshiba, Tokyo), which displayed images on a $512 \times 512$ matrix. The Fisher group ${ }^{6}$ was assessed. The amounts of subarachnoid blood in the interhemispheric fissure, lateral and basal sylvian fissures, and suprasellar, ambient, and quadrigeminal cisterns were evaluated semiquantitatively as scores of 0 to 3 according to the amount of extravasated clot: 0 , nil; 1 , small amount; 2, moderate filling; and 3, complete filling. ${ }^{8)}$ These scores were added to give an estimate of the volume of SAH on admission. The presence of acute hydrocephalus was also evaluated from the CT scan. Hijdra score ${ }^{8}$ on admission and the presence of acute hydrocephalus were evaluated independently by two observers unaware of the medical records. Cerebral angiography was performed in all patients and used to define the location of the aneurysm.

\section{Aneurysm clipping and CSF space irrigation}

Continuous ventricular drainage was used to facilitate aneurysm clipping in six patients with acute hydrocephalus who had clinical deterioration, ventricular enlargement on CT, or large amounts of intraventricular blood. The pterional, inter- 
Table 2 Summary of the characteristics of patients with and without late hydrocephalus after subarachnoid hemorrhage

\begin{tabular}{|c|c|c|c|c|}
\hline & & \multicolumn{2}{|c|}{ Late hydrocephalus } & \multirow{2}{*}{$\mathrm{p}$ Value } \\
\hline & & $+(n=46)$ & $-(n=49)$ & \\
\hline Sex & $\begin{array}{l}\mathrm{M} \\
\mathrm{F}\end{array}$ & $\begin{array}{l}12(26.1) \\
34(73.9)\end{array}$ & $\begin{array}{l}18(36.7) \\
31(63.3)\end{array}$ & 0.265 \\
\hline Age, years & mean \pm SD & $62.5 \pm 13.0$ & $57.3 \pm 13.8$ & 0.0639 \\
\hline Hunt and Hess grade & $\begin{array}{l}\text { I-II } \\
\text { III-V }\end{array}$ & $\begin{array}{l}23(50.0) \\
23(50.0)\end{array}$ & $\begin{array}{l}36(73.5) \\
13(26.5)\end{array}$ & 0.0184 \\
\hline Fisher group & $\begin{array}{l}3 \\
\text { others }\end{array}$ & $\begin{array}{l}32(69.6) \\
14(30.4)\end{array}$ & $\begin{array}{l}18(36.7) \\
31(63.3)\end{array}$ & 0.0014 \\
\hline Presurgery Hijdra score & $\operatorname{mean} \pm \mathrm{SD}$ & $13.7 \pm 5.54$ & $9.35 \pm 6.23$ & 0.0006 \\
\hline Glasgow Coma Scale score & $\begin{array}{l}\leq 8 \\
9-12 \\
13-15\end{array}$ & $\begin{array}{r}9(19.6) \\
4(8.70) \\
33(71.7)\end{array}$ & $\begin{array}{r}3(6.12) \\
5(10.2) \\
41(83.7)\end{array}$ & 0.143 \\
\hline Site of ruptured aneurysm & $\begin{array}{l}\text { ICA } \\
\text { AcomA } \\
\text { ACA } \\
\text { MCA } \\
\text { VBA }\end{array}$ & $\begin{array}{r}11(23.9) \\
18(39.1) \\
3(6.52) \\
11(23.9) \\
3(6.52)\end{array}$ & $\begin{array}{r}15(30.6) \\
13(26.5) \\
4(8.16) \\
15(30.6) \\
2(4.08)\end{array}$ & 0.683 \\
\hline CSF drainage & $\begin{array}{l}\text { non } \\
\text { CVD } \\
\text { CVD + others } \\
\text { others }\end{array}$ & $\begin{array}{r}6(13.0) \\
11(23.9) \\
25(54.3) \\
4(8.70)\end{array}$ & $\begin{array}{r}22(44.9) \\
9(18.4) \\
13(26.5) \\
5(10.2)\end{array}$ & 0.0043 \\
\hline Duration of CSF drainage, days & $\operatorname{mean} \pm \mathrm{SD}$ & $14.5 \pm 8.43$ & $5.22 \pm 6.05$ & $<0.0001$ \\
\hline Neurological deficits at 2 weeks & $\begin{array}{l}+ \\
-\end{array}$ & $\begin{array}{r}44(95.7) \\
2(4.35)\end{array}$ & $\begin{array}{l}17(34.7) \\
32(65.3)\end{array}$ & $<0.0001$ \\
\hline Acute hydrocephalus & $\begin{array}{l}+ \\
-\end{array}$ & $\begin{array}{r}3(6.52) \\
43(93.5)\end{array}$ & $\begin{array}{r}1(2.04) \\
48(98.0)\end{array}$ & 0.277 \\
\hline
\end{tabular}

Figures in parentheses are percentages. ACA: anterior cerebral artery, AcomA: anterior communicating artery, CSF: cerebrospinal fluid, CVD: continuous ventricular drainage, ICA: internal carotid artery, MCA: middle cerebral artery, VBA: vertebrobasilar artery.

hemispheric, lateral suboccipital, and transpetrosal approaches were used as required. The diagnosis of ruptured aneurysm was confirmed intraoperatively in all patients. As much SAH clot was removed intraoperatively as was safely possible to prevent vasospasm. Continuous ventricular drainage and either cisternal drainage or lumbar drainage were also initiated during surgery. Cisternal drains were placed in the prepontine or chiasmal cistern. CSF drainage was intended to reduce intracranial pressure, to induce brain relaxation in the early stage of surgery, and to irrigate the residual subarachnoid and ventricular clots. CSF drainage was discontinued when the symptoms of the patient became stable and the intracranial pressure normalized.

\section{Symptomatic vasospasm}

Symptomatic vasospasm following SAH was defined as significant transient or permanent neurological deterioration, such as hemiparesis or aphasia. Infarction was thought to be due to cerebral vasospasm if the early postoperative CT did not reveal any lesion but CT performed between 1 and 2 weeks later demonstrated cerebral infarction. Possible cerebral vasospasm was identified with transcranial Doppler sonography, cerebral angiography, magnetic resonance (MR) imaging, and/or technetium-99m hexamethyl-propyleneamine oxime in all patients with cerebral infarction on late CT scans. Prophylactic use of calcium channel blocker was not performed. Patients with symptomatic vasospasm were treated with hypertensive-hypervolemic therapy, low-molecular weight dextran, and glycerol. Some patients underwent percutaneous transluminal angioplasty and intraarterial administration of papaverine hydrochloride.

\section{Late hydrocephalus}

The criteria for VP shunting for late hydrocephalus included enlargement of the ventricular system, ${ }^{20,21)}$ periventricular lucency on $\mathrm{CT}$ or periventricular hyperintensity on $\mathrm{T}_{2}$-weighted $\mathrm{MR}$ imaging, and improvement of symptoms after repetitive withdrawal of CSF by lumbar puncture. ${ }^{2,20,22)}$ In this study, patients with late hydrocephalus were defined as those who required VP shunting in the 
subacute and chronic stages after SAH.

\section{Statistical analysis}

Values are expressed as mean \pm SD. Univariate analysis was performed to assess the relationships between various factors: the occurrence of symptomatic vasospasm or late hydrocephalus after SAH was assessed by Student's t-test, the chi-squared test, and the Mann-Whitney test. Some demographic factors and factors statistically associated with occurrence of symptomatic vasospasm or late hydrocephalus were analyzed further by multiple logistic regression analysis. For factors that were independently associated with the occurrence of symptomatic vasospasm or late hydrocephalus after $\mathrm{SAH}$, odds ratios were calculated for interactions between pairs of factors. Interobserver agreement was estimated using the Spearman correlation coefficient for Hijdra score and the kappa coefficient for the presence or absence of acute hydrocephalus on the admission CT scan. Values of $p<0.05$ were considered significant.

\section{Results}

Interobserver agreement for the Hijdra score was very high using the Spearman rank correlation coefficient (correlation coefficient 0.91). Agreement was also very high for the presence or absence of acute hydrocephalus (kappa coefficient 0.96).

Thirty-eight of the 95 patients experienced symptomatic vasospasm after SAH, whereas 57 patients did not. Demographic variables such as sex and age were not related to the occurrence of symptomatic vasospasm by univariate analysis (Table 1). The two groups showed no significant difference in Fisher group or Glasgow Coma Scale score on admission, presence of acute hydrocephalus, site of the rup- tured aneurysm, or route and combination of CSF drainage, although Fisher group and route and combination of CSF drainage tended to differ. However, the total presurgery Hijdra score was greater and duration of CSF drainage longer in the patients with symptomatic vasospasm than in those without symptomatic vasospasm. Further, Hunt and Hess grade was more severe and neurological deficits at 2 weeks after SAH occurred more frequently in the patients with symptomatic vasospasm.

After eliminating closely related variables, the following were adopted as confounders in the logistic regression model for multivariate analysis: age, sex, total presurgery Hijdra score, presence of acute hydrocephalus, and duration of drainage. After adjustment for other variables, there was a significant association between the occurrence of symptomatic vasospasm and greater duration of drainage, with a cut-off value of 11 days (Tables 3 and 4). Although there was no statistical significance, higher Hijdra score trended to be associ-

Table 3 Multiple logistic regression analysis of factors related to occurrence of symptomatic vasospasm after subarachnoid hemorrhage

\begin{tabular}{lccl}
\hline & Adjusted OR & $95 \%$ CI & p Value \\
\hline Age (1 year) & 1 & $0.968-1.04$ & 0.846 \\
Sex (male) & 1.69 & $0.584-4.91$ & 0.332 \\
Presurgery Hijdra score & 0.931 & $0.856-1.01$ & 0.0901 \\
$\quad$ (1 point) & 1.17 & $0.142-9.62$ & 0.885 \\
$\begin{array}{l}\text { Acute hydrocephalus } \\
\text { Drainage duration }\end{array}$ & 0.347 & $0.135-0.889$ & 0.0274 \\
$\quad(\geq 11$ days) & & &
\end{tabular}

CI: confidence interval, OR: odds ratio.

Table 4 Multiple logistic regression analysis of duration of cerebrospinal fluid (CSF) drainage related to occurrence of symptomatic vasospasm and late hydrocephalus

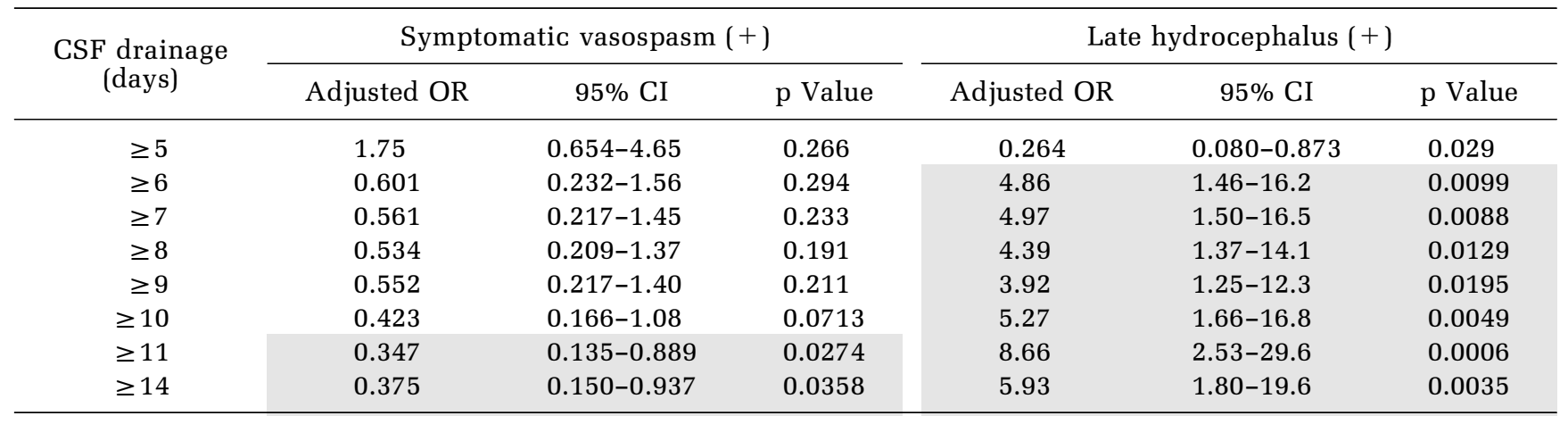

CI: confidence interval, OR: odds ratio. 
Table 5 Multiple logistic regression analysis of factors related to occurrence of late hydrocephalus after subarachnoid hemorrhage

\begin{tabular}{lccc}
\hline & Adjusted OR & $95 \%$ CI & p Value \\
\hline Age (1 year) & 0.996 & $0.949-1.05$ & 0.879 \\
Sex (male) & 1.6 & $0.413-6.17$ & 0.498 \\
$\begin{array}{l}\text { Presurgery Hijdra score } \\
\quad(1 \text { point) }\end{array}$ & 1.04 & $0.937-1.15$ & 0.481 \\
$\begin{array}{l}\text { Neurological deficits } \\
\quad \text { at 2 weeks }\end{array}$ & 34.2 & $6.51-179$ & $<0.0001$ \\
$\begin{array}{l}\text { Acute hydrocephalus } \\
\text { Drainage duration }\end{array}$ & 0.834 & $0.060-11.7$ & 0.893 \\
$\quad$ ( $\geq 6$ days) & 4.86 & $1.46-16.2$ & 0.0099 \\
\hline
\end{tabular}

CI: confidence interval, OR: odds ratio.

ated with symptomatic vasospasm after SAH (Table 3).

Forty-six of the 95 patients underwent VP shunting due to late hydrocephalus. The interval between the onset of SAH and VP shunting was $46.8 \pm 16.9$ days (mean $\pm \mathrm{SD}$ ). The patients with late hydrocephalus included more severe cases according to the Hunt and Hess grade, Fisher group, and Hijdra score (Table 2). CSF drainage was performed more frequently and for longer in the late hydrocephalus group. Neurological deficits at 2 weeks after SAH were also found more often in this group by univariate analysis. Since there were internal correlations between pairs of these variables, multiple logistic regression analysis was used to determine the independent relationships between the occurrence of late hydrocephalus and confounders. After adjustment for other variables, there was a significant association between the occurrence of late hydrocephalus and longer drainage duration, with a cutoff value of 6 days (Tables 4 and 5). Further, neurological deficits at 2 weeks after onset of SAH were found to be a strong independent predictor of late hydrocephalus (Table 5).

The use of vasopressor, low-molecular dextran, or glycerol was more frequent in the patients with symptomatic vasospasm than in those with nonsymptomatic vasospasm (data not shown). This result was due to the protocol for treatment of symptomatic vasospasm.

\section{Discussion}

The present study found that CSF drainage without fibrinolytic agents for $\geq 11$ days can reduce symptomatic vasospasm significantly. Oxyhemoglobin is one of the most important candidate spasmogenic substances. Measurement of sequential changes of oxyhemoglobin concentration showed decreases by day 5 and maintenance at a very low level with urokinase irrigation, but increases peaking on day 9 without urokinase irrigation. ${ }^{13)}$

Our previous report ${ }^{9)}$ and others ${ }^{1,12,15)}$ have indicated that duration of CSF drainage is a statistically significant predictor of late hydrocephalus after SAH. Continuous CSF drainage may promote occlusion of physiological CSF pathways. Therefore, intermittent CSF drainage has been suggested to keep the normal subarachnoid space open, because intermittent CSF pressure is an effective force in preventing the formation of occlusive membranes and clots. ${ }^{11)}$ Our previous study ${ }^{9}$ showed that brain damage due to intraoperative manipulation and cerebral vasospasm is involved in the pathogenesis of late hydrocephalus. Excessive drainage of CSF is related to a greater incidence of cerebral infarction and occurrence of late hydrocephalus. ${ }^{1,12)}$ It is possible that such cerebral infarction is causally related to the occurrence of late hydrocephalus.

Previously, oxyhemoglobin was discharged in considerable amounts via drainage up to day $11 .{ }^{13)}$ This data is compatible with ours. The present study found that CSF drainage without irrigation by fibrinolytic agents cannot prevent both symptomatic vasospasm and late hydrocephalus. CSF drainage up to day 6 prevents only late hydrocephalus, and after day 11 only symptomatic vasospasm, and neither type of event in the intervening period. However, data on the oxyhemoglobin concentration of effluent after urokinase irrigation demonstrates the minimum occurs on day $5,{ }^{13)}$ indicating that effective CSF space irrigation with urokinase can prevent both symptomatic vasospasm and late hydrocephalus in the critical period beginning about 5 days after acute surgery for ruptured aneurysm.

The present study is limited by the small sample size and as a retrospective cohort study. Further study of the validity in a randomized controlled trial is recommended.

\section{References}

1) Auer LM, Schneider GH, Auer T: Computerized tomography and prognosis in early aneurysmal surgery. J Neurosurg 65: 217-221, 1986

2) Black PM: Hydrocephalus and vasospasm after subarachnoid hemorrhage from ruptured intracranial aneurysms. Neurosurgery 18: 12-16, 1986

3) Boullin DJ, Brandt L, Ljunggren B: Vasoconstrictor activity in cerebrospinal fluid from patients subjected to early surgery for ruptured intracranial aneurysms. J Neurosurg 55: 237-245, 1981

4) Findlay JM, Kassell NF, Weir BK, Haley EC Jr, Kongable G, Germanson T, Truskowski L, Alves WM, 
Holness RO, Knuckey NW, Yonas H, Steinberg GK, West M, Winn HR, Ferguson G: A randomized trial of intraoperative, intracisternal tissue plasminogen activator for the prevention of vasospasm. Neurosurgery 37: 168-178, 1995

5) Findlay JM, Weir BKA, Steinke D, Tanabe T, Gordon P, Grace M: Effect of intrathecal thrombolytic therapy on subarachnoid clot and chronic vasospasm in a primate model of SAH. J Neurosurg 69: 723-735, 1988

6) Fisher CM, Kistler JP, Davis JM: Relation of cerebral vasospasm to subarachnoid hemorrhage visualized by computerized tomographic scanning. Neurosurgery 6: 1-9, 1980

7) Higashida RT, Halbach VV, Cahan LD, BranzZawadzki M, Barnwell S, Dowd C, Hieshima GB: Transluminal angioplasty for treatment of intracranial vasospasm. J Neurosurg 71: 648-653, 1989

8) Hijdra A, Brouwers PJ, Vermeulen M, van Gijin J: Grading the amount of blood on computed tomograms after subarachnoid hemorrhage. Stroke 21: 1156-1161, 1990

9) Hirashima Y, Hamada H, Hayashi N, Kuwayama N, Origasa H, Endo S: Independent predictors of late hydrocephalus in patients with aneurysmal subarachnoid hemorrhage - analysis by multivariate logistic regression model. Cerebrovasc Dis 16: 205-210, 2003

10) Hunt WE, Hess RM: Surgical risk as related to time of intervention in the repair of intracranial aneurysms. J Neurosurg 28: 14-19, 1969

11) Kaku Y, Yonekawa Y, Tsukahara T: Superselective intraarterial infusion of papaverine for the treatment of cerebral vasospasm after subarachnoid hemorrhage. J Neurosurg 77: 842-847, 1992

12) Kasuya H, Shimizu T, Kagawa M: The effect of continuous drainage of cerebrospinal fluid in patients with subarachnoid hemorrhage: a retrospective analysis of 108 patients. Neurosurgery 28: 56-59, 1991

13) Kodama N, Sasaki T, Kawakami M, Sato M, Asari J: Cisternal irrigation therapy with urokinase and ascorbic acid for prevention of vasospasm after aneurysmal subarachnoid hemorrhage. Outcome in 217 patients. Surg Neurol 53: 110-118, 2000

14) Ohman J, Heiskanen O: Effect of nimodipine on the outcome of patients after aneurysmal subarachnoid hemorrhage and surgery. J Neurosurg 69: 683-686, 1988

15) Ohwaki K, Yano E, Nakagomi T, Tamura A: Relationship between shunt-dependent hydrocephalus after subarachnoid haemorrhage and duration of cerebrospinal fluid drainage. $\mathrm{Br} J$ Neurosurg 18: 130-134, 2004

16) Osaka K: Prolonged vasospasm produced by the break-down products of erythrocytes. J Neurosurg 47: 403-411, 1977

17) Sasaki T, Kodama N, Kawakami M, Sato M, Asari J, Sakurai Y, Watanabe K, Onuma T, Matsuda T: Urokinase cisternal irrigation therapy for prevention of symptomatic vasospasm after aneurysmal subarachnoid hemorrhage. A study of urokinase concentration and the fibrinolytic system. Stroke 31: 1256-1262, 2000

18) Shibuya M, Asano T, Sasaki Y: Effect of Fasudil HCl, a protein kinase inhibitor, on cerebral vasospasm. Acta Neurochir Suppl 77: 201-204, 2001

19) Teasdale GM, Jannett B: Assessment of coma and impaired consciousness. A practical scale. Lancet 2: 81-84, 1974

20) Vale FL, Bradley EL, Fisher WS III: The relationship of subarachnoid hemorrhage and the need for postoperative shunting. J Neurosurg 86: 462-466, 1997

21) Vermeij FH, Hasan D, Vermeulen M, Tanghe HL, van Gijin J: Predictive factors for deterioration from hydrocephalus after subarachnoid hemorrhage. Neurology 44: 1851-1855, 1994

22) Yoshioka $H$, Inagawa $T$, Tokuda $Y$, Inokuchi $F$ : Chronic hydrocephalus in elderly patients following subarachnoid hemorrhage. Surg Neurol 53: 119-125, 2000

Address reprint requests to: Y. Hirashima, M.D., Department of Neurosurgery, Toyama Medical and Pharmaceutical University, 2630 Sugitani, Toyama, Toyama 930-0194, Japan.

e-mail: yhira@ms.toyama-mpu.ac.jp

\section{Commentary}

Hirashima and colleagues have provided a detailed and rigorous statistical analysis of the duration of cerebrospinal fluid (CSF) drainage and the occurrence of symptomatic vasospasm or the need for ventriculoperitoneal shunting for delayed hydrocephalus. With careful multivariable linear regression methodology, they identified a significant and independent association between symptomatic vasospasm and duration of CSF drainage greater than 11 days, and between delayed CSF shunting and CSF drainage for more than 6 days. This information is useful clinically, in helping the prognosis, especially regarding the need for late shunt.

However, the authors seem to confuse association with causation in the Discussion, and they appear to inappropriately conclude that prolonged CSF drainage may cause vasospasm or the need of shunting. Such a conclusion is unjustified, given that the duration of drainage was not independently assigned in different patient cohorts, and is in fact a dependent variable. Sicker patients received prolonged drainage because they were likely in symptomatic spasm (rather than symptomatic spasm being caused by prolonged drainage), and this agrees fully with the 
cut off date of 11 days. Also, patients prone to hydrocephalus received prolonged drainage, more than 6 days, while drainage was successfully tapered earlier in patients less prone to hydrocephalus. Again, prolonged drainage did not likely cause delayed hydrocephalus, but was associated with it.

Other limitations of this study reflected the different types of CSF drainage, including ventricular and cisternal, and these were not applied uniformly in comparable cases. Indications for and the type of duration of drainage were dictated by the clinical situation, rather than by random assignment, and the study was not designed to tackle the various pathophysiologic hypotheses or the causes and effects of CSF drainage. Still, we concur with the broad conclusion that prolonged CSF drainage does not appear to prevent symptomatic vasospasm or the need of subsequent shunting. Instead, it seems to be an index of disease severity, and hence associated with these respective clinical outcomes. I also agree with the authors that other interventions than CSF drainage are obviously needed to impact on the prevalence of vasospasm and delayed shunting.

Issam A. AwAD, M.D., M.Sc., F.A.C.S., M.A. (Hon.) Professor of Neurosurgery

Evanston Northwestern Healthcare Northwestern University Feinberg School of Medicine Evanston, Illinois, U.S.A.

Cerebral vasospasm after intracranial aneurysm rupture is induced by vasospasmogenic substance produced after the degradation of hemoglobin in the subarachnoid space. Drainage of cerebrospinal fluid (CSF) to remove clot from subarachnoid space after aneurysmal subarachnoid hemorrhage is generally believed to be effective in preventing cerebral vasospasm. However, prolonged CSF drainage increases the risk of infection and intracranial hemorrhage. Excessive CSF drainage may also cause the collapse of physiological CSF pathway and result in late development of hydrocephalus. Thus, to place a CSF drain long enough to eliminate the possibility of the development of vasospasm but not too long to induce shunt dependent hydrocephalus or other complications become a dilemma in clinical setting.

In the present study, the authors try to define the optimal duration of CSF drainage after the surgery of ruptured intracranial aneurysm. Their results indicate that to have significant reduction of the occurrence rate of symptomatic vasospasm, CSF drainage has to be maintained for at least 11 days. On the other hand, maintenance of a CSF drainage for longer than 6 days may significantly associate with the development of late hydrocephalus. The authors suggest that cisternal irrigation with fibrinolytic agents to enhance the dissolution of clot and shorten the duration of CSF drainage needed for eliminate vasospasm may be a solution for the above-mentioned dilemma.

The results of this article can be adopted as a useful clinical guideline in treating the patients with aneurysmal subarachnoid hemorrhage.

Yong-Kwang TU, M.D. Department of Neurosurgery College of Medicine and Hospital National Taiwan University Taipei, Taiwan, R.O.C. 